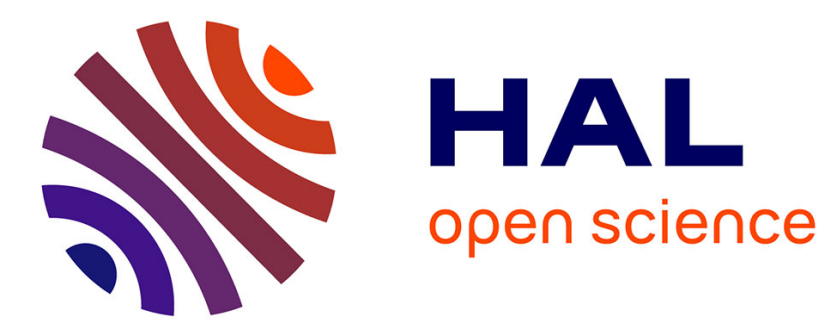

\title{
Ultrasonic Study of the Elastic Behaviour of Zone Refined Single Crystalline Rhodium
}

D. Maurer, R. Heichele, N. Lingg, V. Müller, K. Rieder

\section{To cite this version:}

D. Maurer, R. Heichele, N. Lingg, V. Müller, K. Rieder. Ultrasonic Study of the Elastic Behaviour of Zone Refined Single Crystalline Rhodium. Journal de Physique IV Proceedings, 1996, 06 (C8), pp.C8-535-C8-538. 10.1051/jp4:19968116 . jpa-00254546

\section{HAL Id: jpa-00254546 https://hal.science/jpa-00254546}

Submitted on 1 Jan 1996

HAL is a multi-disciplinary open access archive for the deposit and dissemination of scientific research documents, whether they are published or not. The documents may come from teaching and research institutions in France or abroad, or from public or private research centers.
L'archive ouverte pluridisciplinaire HAL, est destinée au dépôt et à la diffusion de documents scientifiques de niveau recherche, publiés ou non, émanant des établissements d'enseignement et de recherche français ou étrangers, des laboratoires publics ou privés. 


\title{
Ultrasonic Study of the Elastic Behaviour of Zone Refined Single Crystalline Rhodium
}

\author{
D. Maurer, R. Heichele, N. Lingg, V. Müller and K.H. Rieder* \\ Institut für Experimentalphysik III, Universität Augsburg, 86135 Augsburg, Germany \\ * Institut fiir Experimentalphysik, Freie Universität Berlin, 14195 Berlin, Germany
}

\begin{abstract}
We report on temperature dependent sound velocity measurements performed below ambient temperature on a thodium single-crystal of exceptional high purity of about $99.999 \%$. In particular, the excellent acoustic quality of the sample permits a quite accurate determination of the elastic moduli for a sound frequency covering the range between 20 and $150 \mathrm{MHz}$. Primarily our investigations concentrate on the role of the electrons in single-crysalline rhodium which band structure, as speculated, causes a considerable elastic anisotropy and therefore gives rise also to the peculiar mechanical properties commonly known from the poly-crystalline material. A comparative analysis of all stiffness coefficients based on our sound data reveals a strong shear rigidity indicating quite angular dependent crystal forces, which are indeeed not reminiscent of ordinairy metals. Also the temperature dependence studied in detail equally points out that the electronic band structure of modium has to be taken into account for a satisfactory description of the elastic behaviour and cannot be treated properly within the free electron model.
\end{abstract}

\section{INTRODUCTION}

Rhodium is a transition metal with the electron configuration $4 d^{8} 5 s^{1}$ and member of the platinum group. Since pure rhodium is quite corrosion-resistant it finds many applications in thin coating, for crucibles etc.. In addition, it is an important constituent of hard alloys and of catalysts. Solid rhodium possesses a fcc crystal structure like many other metals, but it differs from most of them in being not readily coldworked. The reasons for the difficulty of cold-working are associated with the high rigidity of rhodium but not fully understood till now. It has been suggested, impurities, both metallic and gaseous, play a part and have to be taken into consideration [1]. However, compared with ordinairy metals also pure rhodium possesses a rather low ductility. Therefore it is quite reasonable to assume that these unusual mechanical properties of rhodium are mainly of electronic origin and point to pecularities of the electronic structure, which have to be attributed to the symmetry of the undisturbed crystal lattice.

In a perfect single crystal the elastic moduli are solely determined by the interatomic bonds that is the metallic bond in our case and are thus directly related to the electronic structure of a metal. In general, the determination of stiffness coefficients can be performed ultrasonically with high accuracy. Consequently ultrasonic measurements are quite suitable to investigate the bonding properties in detail. Really surprising, experimental data concerning the elastic behaviour of pure single-crystalline rhodium are hard to find in the technical literature. In particular, there is only one publication found [2], which report on investigations based on ultrasound. However, the data presented there are only of moderate accuracy and were obtained moreover on a specimen of unspecified purity.

\section{EXPERIMENTAL}

The single crystal used in our work was grown from a rhodium-melt of $99.99 \%$ purity as starting material. After zone refining the crystal several times, its total content of metallic impurities should not exceed a concentration of about $10 \mathrm{ppm}$ by weight. A small contribution due to imperfections is further indicated by the rest resistance ratio $\left(R^{300 K} / R^{1 k}\right)$, which has been determined separately on a suitable piece taken from the crystal bulk and which amounts to about 300 even for the unannealed sample. Compared with published data concerning the ratio, which can be achieved [3], the purity of our crystal 
seems to be indeed quite high. In order to get a well shaped sample of high acoustic quality, the single crystal was first spark cutted perpendicular to the [110]-axis, yielding a nearly cylindrical specimen of about $5 \mathrm{~mm}$ in length and $5.5 \mathrm{~mm}$ in diameter. After further grinding and polishing, the end faces become flat and parallel to within $1 \mu \mathrm{m}$ and are oriented within $\pm 1^{\circ}$ to the [110] crystal plane.

\section{RESULTS}

\subsection{Elastic properties}

All experiments done by us were performed with sound propagation along the [110]-axis. This geometry has the great advantage that three independent sound modes (one longitudinal and two transverse) can be excited alternatively, which in the case of cubic symmetry contain the complete information about the crystal elasticity within the framework of linear theory, i.e. the stiffness tensor $\mathbf{C}_{i j}$ of rhodium can be fully determined this way.

The elastic moduli derived from our sound velocity measurements are shown on the lefthand side of Fig.1. All sound velocities obtained are corrected with respect to thermal expansion by means of literature data [4]. Furthermore, the frequency dependence of each sound mode is investigated within the temperature interval of Fig. 1 by exciting different sound frequencies between 20 and $150 \mathrm{MHz}$. In this frequency range our equipment permits measurements with a relative accuracy of about $1 \mathrm{ppm}$ or better. No frequency dependent deviations of the velocity could be detected within $10^{-3}$ - what is close to the limit of absolute resolution. Hence, any non-linearities due to point defects, dislocations etc. are of minor importance, which supports the idea of an almost defect-free crystal as well. Evaluation of our data yields for the independent stiffness coefficients (Voigt notation) in the low temperature limit $(T \rightarrow 0)$ :

$$
C_{11}=41.89 \cdot 10^{10} \mathrm{Nm}^{-2} ; C_{12}=18.85 \cdot 10^{10} \mathrm{Nm}^{-2} ; C_{44}=19.51 \cdot 10^{10} \mathrm{Nm}^{-2} \text {. }
$$

From this the bulk modulos of rhodium can be directly determined:

$$
C_{B}=\frac{1}{3}\left(C_{11}+2 C_{12}\right)=26.43 \cdot 10^{10} \mathrm{Nm}^{-2} \text {. }
$$
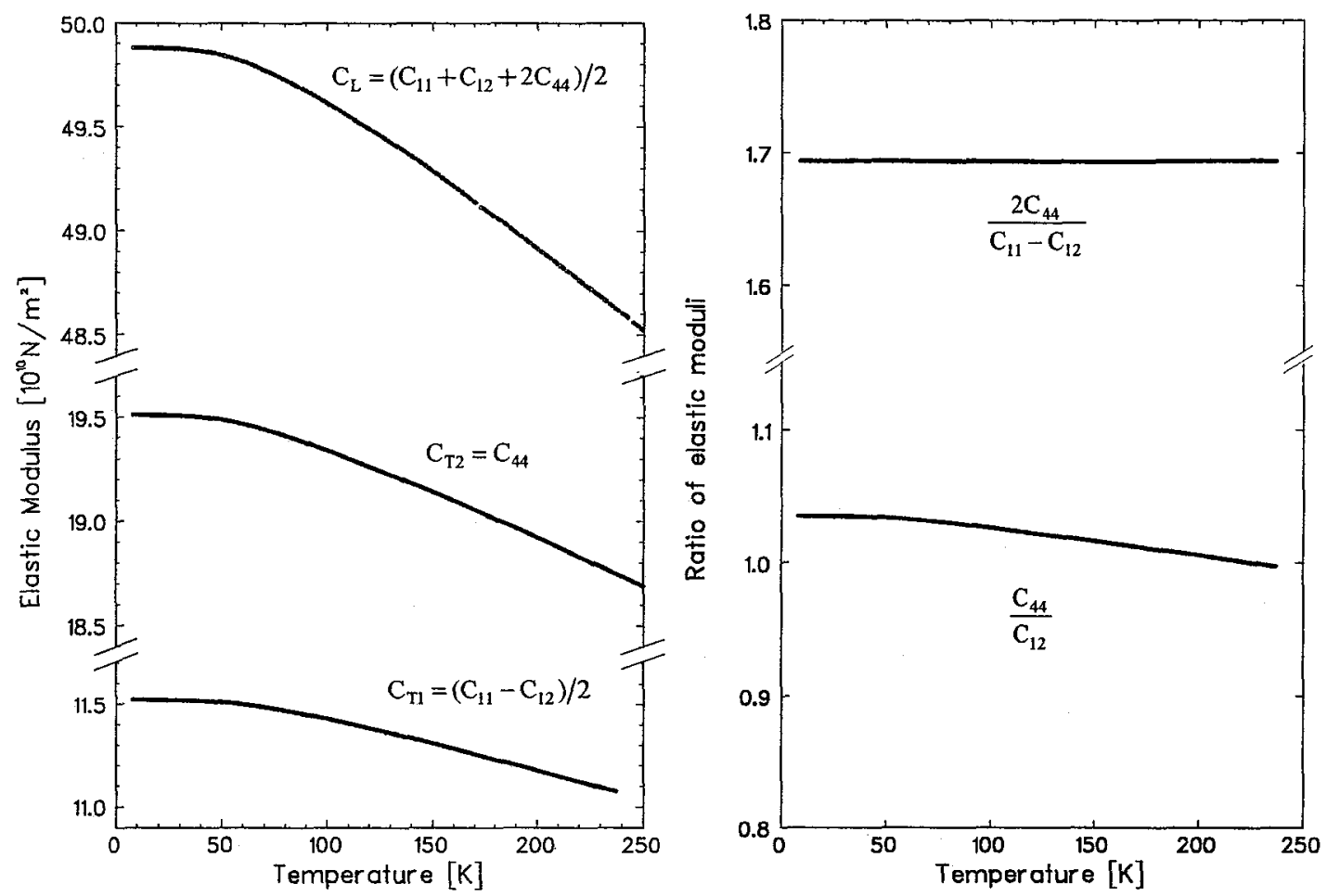

Figure 1: Temperature dependent elastic moduli (left) obtained from sound velocity measurements and ratios characterizing the elastic anisotropy of rhodium (right) derived from the determined stiffness coefficients. 
As is wellknown from the solid state theory of the specific heat, the Debye temperature $\theta$ can be directly calculated from the elastic moduli and need not be determined by any heat capacity measurements. This calculation is of course not easily done exactly, but it can be simplified by various approximation procedures. One of the most powerful and commonly used method, and also prefered by us, is based on tables prepared by de Launay [5]. For rhodium we obtain $\theta=(490 \pm 5) \mathrm{K}$, which is in fair agreement with thermal data even though a comparison suffers from uncertainties due to a quite large scatter of more than $100 \mathrm{~K}$ of the specific heat results published ( see for example ref.[1] ).

Additionally shown in Fig.1 on the right are parameters characterizing the elastic anisotropy of rhodium. The latter, almost independent on temperature, is significantly pronounced yielding an anisotropy factor

$$
2 C_{44} /\left(C_{11}-C_{12}\right)=1.694 \pm 0.001 \text {. }
$$

Apart from the fact that both shear coefficients $\mathbf{C}_{12}$ and $\mathbf{C}_{44}$ are rather large, particular attention should be paid further to their ratio, which in our case points out the validity of the Cauchy relation $C_{12}=C_{44}$ known from lattice theory. This symmetry property follows from supposing central forces between the lattice atoms but is, however, generally not observed in metals due to delocalisation of the bonding electrons. Both size and ratio of the elastic shear moduli are thus quite indicative of strong angular dependent crystal forces, which are reminiscent more of covalent than of metallic single crystals. Hence, the assumption that the low ductility of rhodium could be primarily of electronic origin is indeed quite conclusive in view of this result.

\subsection{Temperature dependence}

At first glance the temperature dependent behaviour of the elastic moduli shows nothing special. Each stiffness coefficient varies almost linear with temperature for $T>100 \mathrm{~K}$ and saturates for $T \rightarrow 0$. Noteworthy, the elastic behaviour at low temperature can be described quite properly by the polynomial

$$
\mathbf{C}=\mathbf{C}_{0}+a \cdot T^{2}+b \cdot T^{4}
$$

Especially for $T<50 \mathrm{~K}$ its accuracy is better than $10^{-5}$ in all cases. The ansatz of Eq.(1) is instructive in so far as it suggests well defined contributions of distinct subsystems, which in the case of a metal can be naturally identified with that one may loosely call its electronic and lattice degree of freedom. According to this the temperature dependence of the elastic moduli should mainly originate in thermal excitations of conduction electrons and phonons respectively. Confined to the low temperature region then the energies corresponding to them can be well approximated by

$$
u_{\mathrm{el}}=\frac{1}{3}\left(\pi k_{\mathrm{B}} T\right)^{2} \cdot \mathrm{N}\left(E_{\mathrm{F}}\right) \text { and } u_{\text {lat }}=\frac{3}{5} \pi^{4} n_{\mathrm{A}} k_{\mathrm{B}} T \cdot(T / \theta)^{3},
$$

where $N\left(E_{F}\right)$ designates the density of states at the Fermi level and $n_{\mathrm{A}}$ the atomic number per volume. Starting from the total internal energy density of the system including the ground state part

$$
u(T)=u_{0}+u_{\mathrm{e}}(T)+u_{\mathrm{latt}}(T)
$$

the stiffness coefficients in the adabatic limit can be directly obtained from

$$
\mathrm{C}_{\mathrm{v}}^{\text {ad. }}=\left.\left\{\partial^{2} u / \partial \varepsilon_{1} \partial \varepsilon_{1}\right\}\right|_{\text {sa const }}
$$

for (symmetrized) strain components $\varepsilon_{1}$. Since already considered in the experimentally determined moduli, the volume dependence due to thermal expansion can be ignored at this point. Now by means of Eq.(4) the temperature behaviour of each subsystem can be analysed separately, when regarding the electronic and the lattice part as independent. Both together then yield a temperature dependence as observed, i.e. in accord to Eq.(1). The individual contributions obtained in this way will be shortly discussed in the following sections.

\subsubsection{Lattice contribution}

Confining oneself to the bulk modulos only, the temperature coefficient of the lattice part can be written

$$
b_{\mathrm{B}}=\frac{2}{3} \pi^{4} n_{\mathrm{A}} k_{\mathrm{B}}\left(\frac{1}{3}+\gamma_{\mathrm{G}}\right) \cdot(\xi / \Theta)^{3} \text { with } \gamma_{\mathrm{G}}=-\left[\partial \ln \Theta / \partial \ln \varepsilon_{\mathrm{B}}\right] \text {. }
$$

The Grüneisen parameter $\gamma_{\mathrm{C}}$ of rhodium amounts to 2.3 [1] and following Eq.(2) the parameter $\xi$ should be equal to one. However, more accurate calculations evidently show that the neclect of anharmonic terms in the expansion of the potential energy of the crystal is not justified in general. Fortunately, consideration of these anharmonic terms in first order simply renomalizes the magnitude of $u_{\text {lett }}$ in Eq.(2) but leaves the $T^{4}$-dependence unchanged [5]. In the frame of quasi-harmonic approximation this again leads to the factor $\xi$ in Eq.(5), which typically varies between 2 and 6 for most metals. The experimental data fitted by Eq.(1) yield $b_{\mathrm{B}}=38 \mathrm{Nm}^{-2} \mathrm{~K}^{4}$. In combination with the determined Debye temperature mentioned above the anharmonic correction thus amounts to $\xi=3.5$ in the case of rhodium. 


\subsubsection{Electronic contribution}

Following Eq.(2) and (4) the temperature coefficient belonging to the conduction electrons becomes

$$
a_{\Gamma}=\frac{1}{3} \pi^{2} k_{\mathrm{B}}^{2} \mathrm{~N}\left(E_{\mathrm{F}}\right) A_{\Gamma}
$$

for a strainfield of symmetry $\Gamma$. Here the parameter $A_{\mathrm{r}}$ depends on both the behaviour of $\mathrm{N}(E)$ near $E_{\mathrm{F}}$ and the coupling strength of the electron states to an acoustic strainfield. $A_{\mathfrak{r}}(E)$ can be written explicitely

$$
A_{\Gamma}=\left\{\left(\frac{\partial \ln N}{\partial \ln E}\right)^{2}-\frac{1}{\mathrm{~N}} \frac{\partial^{2} \mathrm{~N}}{\partial \ln E^{2}}\right\}\left(g_{\Gamma}^{1}\right)^{2}-\frac{\partial \ln N}{\partial \ln E} g_{\Gamma}^{\mathrm{II}} \quad \text { with } \quad g_{\Gamma}^{1}=\frac{1}{E} \frac{d E}{d \varepsilon_{\Gamma}} \quad \text { and } \quad g_{\Gamma}^{\mathrm{II}}=\frac{1}{E} \frac{d^{2} E}{d \varepsilon_{\Gamma}^{2}} .
$$

For free electrons the dimensionless deformation parameters associated with the bulk strain $\varepsilon_{\mathrm{B}}$ are

$$
g_{\mathrm{B}}^{\mathrm{I}}=-\frac{2}{3} \text { and } g_{\mathrm{B}}^{\mathrm{II}}=\frac{10}{9} \text { yielding } A_{\mathrm{B}}^{\mathrm{FE}}=-\frac{2}{9} .
$$

In that case temperature coefficients estimated for conduction electron densities of normal metals achieve some 10 to $100 \mathrm{Nm}^{-2} \mathrm{~K}^{-2}$. This, however, differs much from that found experimentally in rhodium, where $a_{\mathrm{B}}=1.9 \cdot 10^{4} \mathrm{Nm}^{-2} \mathrm{~K}^{-2}$ is obtained ! On the other hand, transition metals can be successfully described within the free electron model by accident only, because their properties are strongly influenced by the existence of narrow d-bands in the vicinity of the Fermi level. In fact, as shown in Fig. 2 the density of states in rhodium is strongly energy dependent near $E_{\mathrm{F}}$. Since especially the parameter $A_{\Gamma}$ is quite sensitiv on $\mathrm{N}\left(E_{\mathrm{F}}\right)$, according to Eq.(6) also the magnitude of the coefficient $a_{\mathrm{B}}$ will depend much on it. Hence, an adequate description of the electronic contribution to the temperature dependent elastic moduli obviously requires that the actual energy dependence of $\mathrm{N}$ has been taken into account.
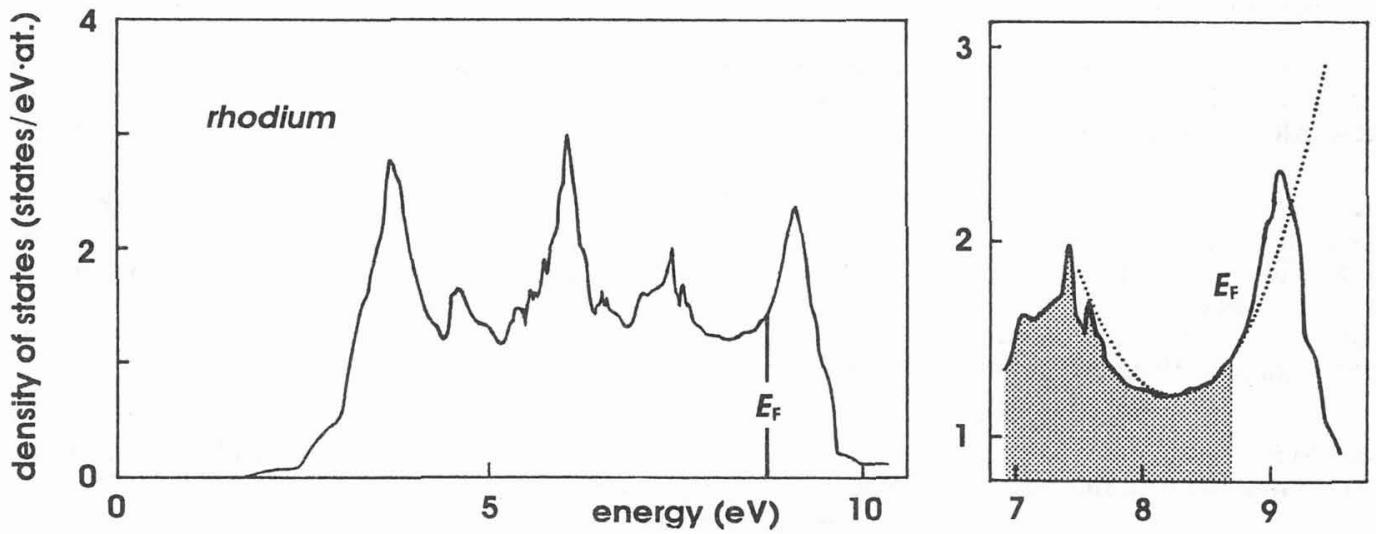

Figure 2: Electronic density of states of rhodium (left) taken from ref.[6] and its approximation near the Fermi level (right).

In order to estimate $a_{\Gamma}$ from $\mathrm{N}(E)$, let us assume for simplicity that the deformation parameters are of same order as in the free electron case. A polynomial approximation of $\mathrm{N}(E)$ in the vicinity of $E_{\mathrm{F}}$

$$
\text { with } \frac{\partial \ln N}{\partial \ln E} \approx 6 \text { and } \frac{1}{N} \frac{\partial^{2} \mathrm{~N}}{\partial \ln E^{2}} \approx 140 \text { then yields } \quad A_{\mathrm{B}} \approx-200
$$

and $a_{\mathrm{B}} \approx 1.7 \cdot 10^{4} \mathrm{Nm}^{-2} \mathrm{~K}^{-2}$ respectively. Though not exact the magnitude estimated this way is nevertheless of the right order and one can conclude that the strong enhancement of $a_{\mathfrak{B}}$ originates primarily in the energy dependence of $N$.

In summary, deriving benefit from the fact that the elastic behaviour of a metal is intimately related to its electronic structure, our investigations essentially point out that the magnitude as well as the temperature dependence of the stiffness coefficients is characterized by the dominant role of d-electrons in single crystalline rhodium. E.g., the pronounced elastic anisotropy of the crystal lattice, reflecting strong angular dependent bonding, confirms the crucial importance of d-like electron states in particular.

\section{References}

[1] Gmelin Handbook of Inorganic and Organometallic Chemistry, $8^{\text {th }}$ Edit., Rhodium Suppl. A1 (1991).

[2] Walker E., Ashkenazi J., and Dacorogna M., Phys. Rev. B 24 (1981) $2254-2256$.

[3] Buchal C., Pobell F., Mueller R., Kubota M., and Owers-Bradley J., Phys. Rev. Lett. 50 (1983) 64-67.

[4] White G.K. and Pawlowicz A.T., J. of Low Temp. Phys. 2 (1970) 631-639.

[5] Leibfried G. and Ludwig W., Solid State Phys. 12 (1961) 275-444.

[6] Moruzzi V.L., Janak J.F., and Williams A.R., Calculated Electronic Properties of Metals (1978). 